\title{
Joining Parents' Bed at Night and Overweight among 2- to 6-Year-Old Children - Results from the 'Healthy Start' Randomized Intervention
}

\author{
Nanna Julie Olsen ${ }^{a} \quad$ Jeanett Friis Rohde ${ }^{a} \quad$ Mina Nicole Händel ${ }^{a}$ \\ Maria Stougaard $^{a}$ Erik Lykke Mortensen ${ }^{b}$ Berit Lilienthal Heitmann ${ }^{a, c, d}$ \\ ${ }^{a}$ Research Unit for Dietary Studies at the Parker Institute, Bispebjerg and Frederiksberg Hospital, \\ the Capital Region, Frederiksberg, Denmark; ${ }^{b}$ Department of Public Health and Center for \\ Healthy Aging, University of Copenhagen, Copenhagen, Denmark; ${ }^{c}$ The Boden Institute of \\ Obesity, Nutrition, Exercise \& Eating Disorders, The University of Sydney, Sydney, Australia, \\ dSection for General Practice, Department of Public Health, University of Copenhagen, \\ Copenhagen, Denmark
}

Keywords

Pediatric obesity $\cdot$ Sleep $\cdot$ Body mass index

\begin{abstract}
Objective: Parental and child co-sleeping has been suggested as a risk factor for short sleep duration and poor sleep quality that may lead to overweight. We examined if joining parent's bed during night was associated with child overweight. Methods: Cross-sectional data from the 'Healthy Start' study including 635 2- to 6-year-old Danish children, who were all considered obesity prone. Of these, 496 children had complete information on BMI and whether the child joined parents' bed during night and if so, how frequently. International cut-offs for overweight according to age and gender were applied, and logistic regression was used to estimate odds ratio (OR) and 95\% Confidence Intervals (Cl). Analyses were adjusted for the child's age and gender, overall family stress, parental educational level, and parental BMI. $\boldsymbol{R e}$ sults: Children who did not join their parent's bed were more likely to be overweight compared to children who did (OR 1.75 (95\% Cl 0.99-3.10)). Children who rarely joined their parents' bed had OR 2.74 of being overweight (95\% Cl 1.01-7.44) compared to children who joined every night. Conclusion: Children who rarely joined parents' bed during night were almost three times more likely to be overweight than those who joined every night.
\end{abstract}

(c) 2018 The Author(s)

Published by S. Karger GmbH, Freiburg 


\section{Introduction}

Childhood overweight (including obesity) is a multifactorial trait, which cannot solely be explained by traditional lifestyle factors, such as too much food and too little physical activity [1]. One alternate modifiable factor that has been found to lead to child overweight is sleep. In this regard, two recent meta-analyses have provided evidence that both short sleep duration and poor sleep quality seem associated with overweight in children and adolescents $[2,3]$. Poor sleep in toddlers and primary school children can impact child growth, and thereby later risk of obesity, via increased cortisol and decreased melatonin levels [4]. These hormonal changes can influence growth and weight in the young child via interactions with insulin, growth hormones, and appetite-regulating hormones like leptin and ghrelin [4, 5]. It has also been suggested that the association between poor sleep and obesity in young children may be mediated by higher energy intake or lower energy expenditure [6,7]. Almost 50\% of parents report perceived sleep problems in toddlers and young children, including the child not sleeping through the night, delay in achieving sleep, and atypical behavioral wakening. Hence, child sleep seems to provide a modifiable and thus promising target for obesity prevention [8]. When intending to improve sleep duration and quality, for example to prevent obesity, it may be considered straightforward to identify factors that are associated with sleep duration and quality, and then aim at modifying them. One of such modifiable factors is 'co-sleeping'. Co-sleeping is an umbrella term that in general is considered to include room sharing, bed sharing, and everything in between $[9,10]$. Co-sleeping between parents and their children is hotly debated, and the literature is often polarized because of challenges in establishing associations between bed sharing and many of the suggested risks or benefits, including the association between bed sharing and Sudden Infant Death Syndrome (SIDS) [10]. In addition to the risk of SIDS, there is evidence to suggest that bed sharing may be associated with adverse outcomes such as short sleep duration and markers of sleep quality such as night-time awakenings and bedtime resistance [10], while the suggested benefits of bed sharing include better mother-child bonding, maternal emotional benefits, and better neuroaffective responses to stress in adulthood [11-13]. However, a substantial portion of the existing literature on bed sharing has dealt with the risk of SIDS, leaving fewer studies and less evidence related to outcomes of co-sleeping later in childhood. Based on the existing evidence, it may be hypothesized that, since co-sleeping is a determinant of short sleep duration and poor sleep quality and short sleep duration and poor sleep quality are determinants for overweight, co-sleeping (including bed sharing) may be a determinant of overweight and obesity. To our knowledge, this hypothesis has only been examined in one previous study in Chinese children aged 3-4 years [14]. This study found a tendency that children who co-slept were less likely to be overweight, although this result was not significant [14]. The purpose of our study was therefore to examine whether overweight and obesity in obesityprone Danish children aged 2-6 years was related to whether or not the children joined their parents' bed during night.

\section{Material and Methods}

\section{Study Sample}

Cross-sectional data derived from the baseline examination of the intervention study 'Healthy Start' ('Sund Start') which was conducted between 2009 and 2011. The intervention aimed at preventing overweight over the subsequent 15 months among Danish children aged 2-6 years, who were classified as predisposed to overweight based on a either being born with a high birth weight $(>4,000 \mathrm{~g})$, having a mother who was overweight prior to pregnancy (BMI $\geq 28 \mathrm{~kg} / \mathrm{m}^{2}$ ), or having a mother with low socioeconomic status (educational level $\leq 10$ years, subgroup only). Information on birth weight and maternal pre-pregnancy BMI 
of all children born in 11 municipalities from the greater Copenhagen area between 2004 and 2007 was obtained from the Danish National Birth Register. In a subgroup, information on maternal educational level was obtained from administrative birth forms completed at the time of birth by hospital personnel. After the selection process, children were identified using their Central Person Registration number. Children were subsequently excluded if they had protection from participation in statistical and scientific surveys, had no permanent address, lived in an orphanage, had unknown life status, had emigrated or moved to another municipality than where they were born. All children who were eligible for inclusion were randomized into an intervention group or a control group and invited to participate in the Healthy Start project. A total of 635 children participated in the baseline examination. Data in the present study is from the baseline examination and include 496 children with complete information on BMI and whether the child entered parents' bed during night and if so, how frequently. The methodology and participation flow charts in the Healthy Start study has previously been described in more detail [15].

\section{Weight Outcomes}

Body height to the nearest $0.1 \mathrm{~cm}$ was measured with a stature meter (Soehnle 5002 or Charter ch200P). Body weight to the nearest $0.1 \mathrm{~kg}$ was measured with a mechanical weight or beam-scale type weight (Tanita BWB-800 or SV-SECA 710). All anthropometric measurements were obtained by trained personnel. BMI was calculated based on the height and weight measurements. The international cut-offs developed by the International Obesity Task Force were used to classify the children into normal-weight or overweight outcome categories, taking age and gender into account [16]. The 'overweight' category also included children who were obese, and the 'normal-weight' category also included children who were underweight.

Parents completed a questionnaire about factors such as meal habits, physical activity, sleep habits, and stress in the family. Except gender and age, all variables described below were obtained from this questionnaire.

\section{Sleep Habits}

To obtain information on bed sharing, parents were asked a general question on whether their child joined their bed during night ('yes' or 'no'). The question was phrased (in Danish) to indicate an active decision, contrasting the child already starting the night in the parents' bed.

If a parent answered 'yes', an additional question asked how often the child joined the parents' bed during night ('Less than once a month', '1-3 times per month', '1-3 times per week', '4-6 times per week', 'Every night'). For children joining the parents' bed never or less than once a month, a 'Rarely' category was used in the frequency analyses.

Parents were also asked how they would classify their child's sleep; the response options to this question were 'The child sleeps calmly all night', 'The child sleeps a bit disturbed', 'The child sleeps disturbed with occasional awakenings', and 'The child sleeps disturbed with several awakenings each night'. This variable was included in a separate statistical model as a potential confounder of the effects of bed sharing.

Finally, prior to completing the questionnaire, the parents were asked to complete a 6-day sleep diary on the bedtimes (fell asleep and woke up) during the week. Information from the sleep diary was used to calculate the mean sleep duration (in minutes) over 6 days.

\section{Confounding Variables}

Self-reported heights and weights of both parents were obtained, and parental BMI was calculated.

Parental highest level of completed education was reported in 9 categories. Of these categories, 8 were recoded into 3 levels; low educational level ('primary and lower secondary school', 'upper secondary', 'one or more short courses (semi-skilled worker courses)', or 'skilled worker'), medium educational level ('shortterm further education ( $<3$ years)', 'medium-term further education (3-4 years)'), and high educational level ('long-term further education ( $>4$ years)', 'research worker level'). Responses in the 9th category were not recoded, as they included types of education, which were not possible to classify according to the originally reported categories (e.g. education completed in foreign countries).

Information on the child's exact age was obtained by subtracting the date of the baseline health examination from the date of birth (obtained from the Danish National Birth register). Information on gender was obtained from the Danish National Birth Register.

The Parenting Stress Index (PSI) is a self-report inventory designed to measure parental experience of stress in the parent-child relation [17]. Ten questions from the Swedish version of PSI were modified and applied to assess the parents' perceptions of the overall stress level in the family. Each question in the PSI 
was scored between 0 and 2 (with 0 being the best score and 2 being the worst), according to its estimated indication of an overall stress level. Analysis of inter-correlations among the 10 questions and a principal component analysis suggested that 9 of the 10 questions could be added together to get a score for the overall family stress level. This score was further recoded into tertiles.

The Danish version of the Strengths and Difficulties Questionnaire (SDQ) was used as an indicator of the child's overall stress level. The SDQ asks about 25 attributes, some positive and others negative. The SDQ Total Difficulties (SDQ-TD) score was calculated for each child, based on scoring syntax available from the SDQ webpage [18]. Like the PSI score, the total SDQ-TD score was recoded into tertiles.

\section{Statistical Methods}

Odds ratios (OR) and 95\% confidence intervals (CI) were estimated from logistic regression analyses. The logistic regression analyses were performed in two steps:

The crude model included only the exposure and the outcome with no adjustment for confounding variables, while the adjusted model adjusted for age, gender, parental educational level, PSI score, and parental BMI. Finally, separate models additionally adjusting for SDQ-TD score and for the quality of the child's sleep (calm or disturbed) were analyzed.

All analyses were performed in SAS 9.3, using the Proc Genmod procedure.

\section{Ethics}

The Scientific Ethical Committee of the Capital Region in Denmark decided that, according to Section 2.-(1) of the Danish Act on a Bioethics Committee System and the Processing of Bioethics Projects, the project was defined not to be a bioethics project and, consequently, did not need approval from the Danish Bioethics Committee (journal number H-A-2007-0019). The Danish Data Protection Agency approved of the study (journal number: 2015-41-3937). Written informed consent to use the collected data for research purposes was obtained from all participants' parents.

\section{Results}

Table 1 shows characteristics of children and parents stratified by whether children joined their parents' bed or not. Children who joined their parents' bed had a lower mean BMI and were older than children who did not. In addition, differences in the proportion of normalweight versus overweight children were found, with more overweight children not joining their parents' bed. A chi-square analysis showed a significant association between joining parents' bed and maternal educational level, with relatively fewer children with maternal high educational level among those who joined their parents' bed. An association between parental perception of the child's sleep and bed joining was furthermore observed, with relatively more children with disturbed sleep among those who joined parents' bed. Finally, children who joined parents' bed had significantly shorter average sleep duration as calculated from the 6-days sleep diaries.

Those children who did not join their parents' bed had almost doubled odds of being overweight compared to those children who did (OR 1.73, 95\% CI 1.06-2.84) (table 2). This difference remained, but the associations became insignificant in the adjusted model (OR 1.75 (95\% CI 0.99-3.10)) (table 2). Furthermore, there was a tendency that the OR of being overweight was lower with increasing frequency of joining parents' bed (table 3). Indeed, compared to children who joined their parents' bed every night, children who rarely joined were almost three times more likely to be overweight, also after adjusting for confounders (OR 2.74, 95\% CI 1.01-7.44) (table 3). There was no interaction between the frequency of joining parents' bed and gender ( $p=0.67$, data not shown) or age $(p=0.36$, data not shown) of the child.

A sensitivity analysis was conducted, adding SDQ-TD score to the adjusted model. In this model the odds for being overweight in relation to joining parents' bed every night compared 
Olsen et al.: Joining Parents' Bed at Night and Overweight among 2- to 6-Year-Old Children - Results from the 'Healthy Start' Randomized Intervention

Table 1. Characteristics by whether or not the child join the parents' bed

\begin{tabular}{|c|c|c|c|c|}
\hline & \multirow[b]{2}{*}{$\mathrm{n}$} & \multicolumn{2}{|c|}{ Does your child join your bed during night? } & \multirow[b]{2}{*}{$\begin{array}{l}\text { p-value, t-tests } \\
\text { or } \chi^{2}\end{array}$} \\
\hline & & $\begin{array}{l}\text { no }(\mathrm{n}=186) \\
\text { mean }(\mathrm{SD})\end{array}$ & $\begin{array}{l}\text { yes }(n=401) \\
\text { mean }(S D)\end{array}$ & \\
\hline Age, years & 587 & $3.8(1.18)$ & $4.1(1.03)$ & $0.02 * \mathrm{~A}$ \\
\hline BMI, $\mathrm{kg} / \mathrm{m}^{2}$ & 587 & $16.5(1.29)$ & $16.2(1.24)$ & $0.03^{* \mathrm{~A}}$ \\
\hline SDQ-TD score, points & 582 & $7.0(3.99)$ & $6.6(3.83)$ & $0.26^{\mathrm{A}}$ \\
\hline PSI score, points & 542 & $12.3(2.38)$ & $12.5(2.33)$ & $0.43^{\mathrm{A}}$ \\
\hline \multirow[t]{2}{*}{ Mean sleep duration, min } & 552 & $650(39.49)$ & $642(35.89)$ & \multirow[t]{2}{*}{$0.02 * \mathrm{~A}$} \\
\hline & & $\%(n)$ & $\%(\mathrm{n})$ & \\
\hline Normal weight / overweight (including obese), $\mathrm{n}$ & 587 & $83 \%(154) / 17 \%(32)$ & $89 \%(358) / 11 \%(43)$ & $0.03^{* \mathrm{~B}}$ \\
\hline Maternal educational level & 586 & & & \\
\hline High & & $31 \%(57)$ & $22 \%(86)$ & \\
\hline Medium & & $45 \%(83)$ & $52 \%(210)$ & \\
\hline Low & & $24 \%(45)$ & $26 \%(105)$ & $0.05^{* B}$ \\
\hline Paternal educational level & 567 & & & \\
\hline High & & $28 \%(49)$ & $23 \%(90)$ & \\
\hline Medium & & $33 \%(58)$ & $39 \%(153)$ & $0.27^{\mathrm{B}}$ \\
\hline Low & & $40 \%(71)$ & $38 \%(146)$ & \\
\hline Parental perception of child sleep & 584 & & & $<0.0001^{* B}$ \\
\hline Calm & & $76 \%(141)$ & $55 \%(218)$ & \\
\hline Slightly disturbed & & $16 \%(29)$ & $34 \%(136)$ & \\
\hline Disturbed with occasional awakenings & & $8 \%(14)$ & $10 \%(38)$ & \\
\hline Very disturbed with frequent awakenings & & $0 \%(1)$ & $0 \%(7)$ & \\
\hline
\end{tabular}

Table 2. Joining the parents' bed and the Odds (OR) of child being overweight

\begin{tabular}{|c|c|c|c|c|c|c|}
\hline & \multicolumn{6}{|c|}{ Does your child join your bed during night? } \\
\hline & \multicolumn{3}{|c|}{ crude model } & \multicolumn{3}{|c|}{ adjusted model ${ }^{1}$} \\
\hline & $\mathrm{n}$ & OR & $95 \% \mathrm{CI}$ & $\mathrm{n}$ & OR & $95 \% \mathrm{CI}$ \\
\hline Total & 587 & & & 498 & & \\
\hline Yes & 401 & 1.00 & - & 341 & 1.00 & - \\
\hline No & 186 & 1.73 & $1.06-2.84 *$ & 157 & 1.75 & $0.99-3.10$ \\
\hline
\end{tabular}

${ }^{1}$ Adjusted for gender, age, parental BMI, PSI-score, and parental educational level.

*Statistical significance.

to never / almost never was strengthened, suggesting that, rather than acting as an intermediate variable potentially explaining why those children who did not join their parents' bed were more obese than children who did, the SDQ-TD score confounded the association and an even stronger odds of overweight was found independently of child's overall stress level (OR 3.68, 95\% CI 1.22-11.04, data not shown).

Additional adjustments of model 1 for parental perceptions of whether the child slept calmly or disturbed gave essentially similar estimates, although the results became insignif- 
Olsen et al.: Joining Parents' Bed at Night and Overweight among 2- to 6-Year-Old Children - Results from the 'Healthy Start' Randomized Intervention

Table 3. Frequency of joining the parents' bed and the Odds (OR) of child being overweight

\begin{tabular}{|c|c|c|c|c|c|c|}
\hline & \multicolumn{6}{|c|}{ How often does your child join your bed during night? } \\
\hline & \multicolumn{3}{|c|}{ crude model } & \multicolumn{3}{|c|}{ adjusted model ${ }^{1}$} \\
\hline & $\mathrm{n}$ & $\mathrm{OR}$ & $95 \%$ CI & $\mathrm{n}$ & OR & $95 \%$ CI \\
\hline Total & 584 & & & 496 & & \\
\hline Every night & 87 & 1.00 & - & 72 & 1.00 & - \\
\hline 4-6 times per week & 92 & 1.39 & $0.51-3.84$ & 79 & 1.67 & $0.52-5.34$ \\
\hline 1-3 times per week & 94 & 1.36 & $0.49-3.75$ & 83 & 1.68 & $0.53-5.38$ \\
\hline 1-3 times per month & 88 & 1.47 & $0.53-4.04$ & 74 & 1.25 & $0.36-4.35$ \\
\hline Rarely & 223 & 2.35 & $1.01-5.48^{*}$ & 188 & 2.74 & $1.01-7.44^{*}$ \\
\hline
\end{tabular}

${ }^{1}$ Adjusted for gender, age, parental BMI, PSI-score, and parental educational level.

*Statistical significance.

icant (OR 2.66, 95\% CI 0.96-7.34, data not shown). Finally, adjusting model 1 for mean sleep duration attenuated the estimate slightly (OR 2.26, 95\% CI 0.82-6.26, data not shown), and the association became insignificant.

\section{Discussion}

We found that children who joined their parent's bed were leaner than children who did not. We also found that those children who rarely joined their parents' bed had the highest odds of being overweight. The associations did not seem to depend on the children's sleep quality or duration and was independent of behavior problems and child's overall stress level as reflected in analyses that included the SDQ-TD score.

Children's bed sharing with parents could reflect various dynamics; a review from 2011 found that, especially in Caucasian families, bed sharing could be reactions to stressors in the child such as bullying, inter-parental conflict, divorce, or general (in-)stability of the home environment $[11,19]$. Stressors in this regard were not limited to family stress but could also include issues such as fear of darkness, recently watching a scary or violent movie, mis-interpretation of an object in darkness as a demon etc. [11]. In addition, separation anxiety as well as factors related to the child's sleep such as nightmares have also been found to predict bed sharing $[20,21]$. A recent review, using a narrative synthesis approach, found that breastfeeding, emotional comforting for mother or infant, better/more sleep, monitoring of the child as well as promotion of bonding between mother and infant were among the most commonly cited reasons for parents choosing bed sharing [13]. Because of the diversity in the dynamics reflected by bed sharing, hypotheses about the mechanisms of the link between bed sharing and overweight beforehand are strictly speculative but may be sought after in the suggested benefits of bed sharing that also have been found to be protective against overweight. In addition, disturbed sleep/wake patterns affecting the leptin circadian rhythm has been suggested as a potential mediator, since poor sleep has been found to systematically decrease leptin levels $[22,23]$. Recent research has also suggested an association between the stress hormone cortisol and overweight [24-26]; in this regard, one prospective study conducted among infants recently found that solitary sleeping in the first month of life was associated with increased cortisol reactivity to mild stressors [27].

Our results are basically similar to the results of the only previously published study on bed sharing and overweight conducted among Chinese children aged 3-4 years. In addition 
our results were also statistically significant [14], which may be attributed to the slightly older age of the children in our study, and/or to the fact that our study participants were all obesity-prone.

Our results add to the present knowledge, suggesting a link between poor sleep and overweight, and may be considered important in relation to future designs of overweight prevention strategies. As described in the introduction, recent reviews have concluded that short sleep duration and poor sleep quality seems associated with childhood overweight and obesity [2,3]. However, so far only few studies have intervened on child sleep habits in relation to risk of obesity development [28-30], leaving rather little experience and inspiration from previous studies when designing a new intervention. A possible way to overcome this would be to examine the upstream factors related to short sleep duration and poor sleep quality, and to assume that a change in these factors may improve both sleep habits and prevent obesity. On the other hand, factors associated with short sleep duration and poor sleep quality such as parental attendance at bedtime and being allowed to enter parents' bed during night $[31,32]$ may at the same time be indicators of e.g. parental attachment. Indeed, most behavioral interventions designed to improve infant sleep were focused on reducing and withdrawing parental involvement, in order to encourage development of self-regulating abilities, which have raised concerns that child-parent attachments may be compromised [31]. Even though no evidence for such adverse effects has been found hitherto [31], it may be argued that factors correlating with sleep duration and quality should be intervened on with more caution if they are considered to be related to the parent-child relationship, as compared with factors such as screen time or physical activity level. In this regard, it has been suggested that the association between short sleep duration and overweight cannot be explained by sociodemographic factors or unhealthy dietary factors but is determined by factors in the physical and sociocultural home environment [33]. Hence, additional studies within this area of childhood obesity research are thought to be important.

A few limitations should be noted; our data is cross-sectional, and, hence, we cannot conclude anything about causality. In line with this, we cannot exclude that our results reflect that overweight children are less likely to be allowed to join their parents' bed at night than the normal-weight children, although this is not considered very likely. Another limitation may be that information on whether joining the parents' bed was reactive or planned and on how frequently during the one night the child attempted to join their parents' bed is not available. This information could have enabled a distinction between those children attempting to join the parents' bed and being rejected, those children not attempting to join the parents' bed (and hence not rejected), and those children attempting and being allowed to join their parents' bed, which may have provided valuable information for the interpretation of the results. In addition, information on parent-child attachment (e.g. parenting style) was not obtained but could have been either confounding or mediating our associations and, thus, contributing to possibly explaining some of the mechanism behind our results.

It should of course be noted, that the present group of children were all considered to be predisposed to future overweight and obesity. Therefore, the associations observed in the present study may differ from the results that may be obtained in a general population sample or in a sample selected based on other predisposing factors than those in the present study (e.g. low birth weight, early adiposity rebound). Hence, generalization of these results should be done with caution. Nonetheless, more research looking into the determinants of short sleep duration and poor sleep quality in relation to development of overweight and obesity is needed. 


\section{Conclusion}

We found that young children aged 2-6 years who joined their parents' bed during night were less overweight than children that did not join. Indeed, children who rarely joined their parents' bed had almost threefold increased odds of being overweight compared to children who joined every night. Our results suggest that, even if children who joined their parents' bed may have had a shorter or more fragmented sleep, they did not appear to be more overweight.

\section{Acknowledgments}

We wish to thank all families who participated in the 'Healthy Start' project, and all employees involved in the data collection.

\section{Funding Source}

The Healthy Start study was funded by the Tryg Foundation (grant: 7984-07, 7106-09, and 7-100330), the Danish Medical Research Council (grant: 271-07-0281), and the Health Insurance Foundation (grant: 2008B101).

\section{Clinical Trial Registration}

'The Healthy Start Project: Primary Prevention of Overweight in Preschool Children Predisposed to Future Overweight', ID NCT01583335, https://clinicaltrials.gov/ct2/show $/$ NCT01583335?term = healthy+start+study\&rank $=3$.

\section{Disclosure Statement}

The authors have no conflicts of interest relevant to this article to disclose. No study sponsors had a role in study design, the collection, analysis and interpretation of data, the writing of the report, and the decision to submit the paper for publication.

\section{References}

1 Van Cauter E, Knutson KL: Sleep and the epidemic of obesity in children and adults. Eur J Endocrinol 2008; 159(suppl 1):S59-S66.

-2 Fatima Y, Doi SA, Mamun AA: Sleep quality and obesity in young subjects: a meta-analysis. Obes Rev 2016;17: 1154-1166.

-3 Fatima Y, Doi SA, Mamun AA: Longitudinal impact of sleep on overweight and obesity in children and adolescents: a systematic review and bias-adjusted meta-analysis. Obes Rev 2015;16:137-149.

4 Copinschi G, Leproult R, Spiegel K: The important role of sleep in metabolism. Front Horm Res 2014;42:59-72.

-5 Taheri S, Lin L, Austin D, Young T, Mignot E: Short sleep duration is associated with reduced leptin, elevated ghrelin, and increased body mass index. PLoS Med 2004;1:e62.

-6 Fisher A, McDonald L, van Jaarsveld CH, Llewellyn C, Fildes A, Schrempft S, Wardle J: Sleep and energy intake in early childhood. Int J Obes (Lond) 2014;38:926-929.

7 Mindell JA, Kuhn B, Lewin DS, Meltzer LJ, Sadeh A: Behavioral treatment of bedtime problems and night wakings in infants and young children. Sleep 2006;29:1263-1276.

8 Tham EK, Schneider N, Broekman BF: Infant sleep and its relation with cognition and growth: a narrative review. Nat Sci Sleep 2017;9:135-149.

-9 Jiang Y, Chen W, Spruyt K, Sun W, Wang Y, Li S, Shen X, Wang G, Jiang F: Bed-sharing and related factors in early adolescents. Sleep Med 2016;17:75-80. 
Olsen et al.: Joining Parents' Bed at Night and Overweight among 2- to 6-Year-Old

Children - Results from the 'Healthy Start' Randomized Intervention

10 Mileva-Seitz VR, Bakermans-Kranenburg MJ, Battaini C, Luijk MP: Parent-child bed-sharing: the good, the bad, and the burden of evidence. Sleep Med Rev 2016;32:4-27.

11 Jain S, Romack R, Jain R: Bed sharing in school-age children - clinical and social implications. J Child Adolesc Psychiatr Nurs 2011;24:185-189.

12 Sobralske MC, Gruber ME: Risks and benefits of parent/child bed sharing. J Am Acad Nurse Pract 2009;21: 474-479.

13 Ward TC: Reasons for mother-infant bed-sharing: a systematic narrative synthesis of the literature and implications for future research. Matern Child Health J 2015;19:675-690.

-14 Jiang F, Zhu S, Yan C, Jin X, Bandla H, Shen X: Sleep and obesity in preschool children. J Pediatr 2009;154:814818.

15 Olsen NJ, Buch-Andersen T, Handel MN, Ostergaard LM, Pedersen J, Seeger C, Stougaard M, Traerup M, Livemore K, Mortensen EL, Holst C, Heitmann BL: The Healthy Start project: a randomized, controlled intervention to prevent overweight among normal weight, preschool children at high risk of future overweight. BMC Public Health 2012;12:590.

16 Cole TJ, Bellizzi MC, Flegal KM, Dietz WH: Establishing a standard definition for child overweight and obesity worldwide: international survey. BMJ 2000;320:1240-1243.

17 Ostberg M, Hagekull B, Wettergren S: A measure of parental stress in mothers with small children: dimensionality, stability and validity. Scand J Psychol 1997;38:199-208.

18 SDQ. Information for researchers and professionals about the Strengths \& Difficulties Questionnaires. www. sdqinfo.com/py/sdqinfo/b3.py?language=Danish (last accessed September 26, 2018).

19 Schwichtenberg AJ, Goodlin-Jones B: Causes and correlates of frequent night awakenings in early childhood. Int Rev Neurobiol 2010;93:177-191.

20 BaHammam A, Alameri H, Hersi A: Cosleeping and its correlates in Saudi school-aged children. Int J Behav Med 2008;15:336-340.

21 Jenni OG, Fuhrer HZ, Iglowstein I, Molinari L, Largo RH: A longitudinal study of bed sharing and sleep problems among Swiss children in the first 10 years of life. Pediatrics 2005;115:233-240.

22 Pervanidou P, Chrousos GP: Metabolic consequences of stress during childhood and adolescence. Metabolism 2012;61:611-619.

-23 Pervanidou P, Chrousos GP: Stress and obesity/metabolic syndrome in childhood and adolescence. Int J Pediatr Obes 2011;6(suppl 1):21-28.

-24 Miller AL, Clifford C, Sturza J, Rosenblum K, Vazquez DM, Kaciroti N, Lumeng JC: Blunted cortisol response to stress is associated with higher body mass index in low-income preschool-aged children. Psychoneuroendocrinology 2013;38:2611-2617.

25 Miller AL, Sturza J, Rosenblum K, Vazquez DM, Kaciroti N, Lumeng JC: Salivary alpha amylase diurnal pattern and stress response are associated with body mass index in low-income preschool-aged children. Psychoneuroendocrinology 2015;53:40-48.

-26 Champaneri S, Xu X, Carnethon MR, Bertoni AG, Seeman T, DeSantis AS, Diez RA, Shrager S, Golden SH: Diurnal salivary cortisol is associated with body mass index and waist circumference: the Multiethnic Study of Atherosclerosis. Obesity (Silver Spring) 2013;21:E56-E63.

27 Tollenaar MS, Beijers R, Jansen J, Riksen-Walraven JM, de Weerth C: Solitary sleeping in young infants is associated with heightened cortisol reactivity to a bathing session but not to a vaccination. Psychoneuroendocrinology 2012;37:167-177.

-28 Wake M, Price A, Clifford S, Ukoumunne OC, Hiscock H: Does an intervention that improves infant sleep also improve overweight at age 6? Follow-up of a randomised trial. Arch Dis Child 2011;96:526-532.

-29 Puder JJ, Marques-Vidal P, Schindler C, Zahner L, Niederer I, Burgi F, Ebenegger V, Nydegger A, Kriemler S: Effect of multidimensional lifestyle intervention on fitness and adiposity in predominantly migrant preschool children (Ballabeina): cluster randomised controlled trial. BMJ 2011;343:d6195.

-30 Paul IM, Savage JS, Anzman SL, Beiler JS, Marini ME, Stokes JL, Birch LL: Preventing obesity during infancy: a pilot study. Obesity (Silver Spring) 2011;19:353-361.

-31 Sadeh A, Tikotzky L, Scher A: Parenting and infant sleep. Sleep Med Rev 2010;14:89-96.

-32 Touchette E, Petit D, Tremblay RE, Montplaisir JY: Risk factors and consequences of early childhood dyssomnias: new perspectives. Sleep Med Rev 2009;13:355-361.

-33 de JE, Stocks T, Visscher TL, HiraSing RA, Seidell JC, Renders CM: Association between sleep duration and overweight: the importance of parenting. Int J Obes (Lond) 2012;36:1278-1284. 